\title{
UN TEOREMA DE DESINGULARIZACIÓN
}

\author{
Renato Benazic Tomé ${ }^{1}$
}

\begin{abstract}
En el presente trabajo, planteamos y resolvemos el problema de desingularización para campos vectoriales holomorfos definidos en una variedad analítica compleja de dimension tres, los cuales presentan una singularidad absolutamente aislada
\end{abstract}

\section{INTRODUCCIÓN}

Sea $\mathcal{M}^{n}$ una variedad analítica compleja de dimension $n$ y consideremos en ella una foliación analítica singular por curvas. Esto significa que en cualquier punto $p \in \mathcal{M}^{n}$ la foliación es generada por el campo vectorial holomorfo

$$
Z=\sum_{i=1}^{n} Z_{i} \frac{\partial}{\partial z_{i}}
$$

en donde $Z_{1}, Z_{2}, \ldots, Z_{n} \in \mathcal{O}_{n, p}$ (aquí $\mathcal{O}_{n, p}$ es el anillo de gérmenes de las funciones analíticas en $p) ;$ y m.c.d. $\left(Z_{1}, Z_{2}, \ldots, Z_{n}\right)=1$. En lo sucesivo, denotaremos por $\mathcal{F}_{Z}$ a esta foliación, diremos que el campo $Z$ genera la foliación $\mathcal{F}_{7}$ y las funciones $Z_{i}$ serán llamadas componentes de $Z$. El lector interesado en conocer detalles de la teoría de funciones analíticas de varias complejas, deberá consultar [10] y para los que deseen profundizar en la teoría de las variedades analíticas complejas, recomendamos [14].

Sea $p \in \mathcal{M}^{n}$ y consideremos una carta $(U, \phi)$ de $\mathcal{M}^{n}$ alrededor del punto $p$ tal que $\phi(p)=0 \in \mathbb{C}^{n}$, claramente $Z_{i} \circ \phi^{-1}$ es una función analítica de varias variables complejas definida en una vecindad del origen y por lo tanto, ella tiene un desarrollo en series de potencias

$$
Z_{i} \circ \phi^{-1}=\sum_{k \geq 0} Z_{i}^{k}, \quad 1 \leq i \leq n
$$

\footnotetext{
${ }^{1}$ Instituto de Matemática y Ciencias Afines (IMCA) y Universidad Nacional Mayor de San Marcos.
} 
donde los $Z_{i}^{k}$ son polinomios homogéneos de grado $k$ en $n$ variables complejas. El orden de $Z_{i} \circ \phi^{-1}$ en el $0 \in \mathbb{C}^{n}$ es, por definición, el menor número entero $\nu_{i}$ tal que $Z_{i}^{k} \equiv 0$, para todo $k<\nu_{i}$ y $Z_{i}^{\nu_{i}} \not \equiv 0$. No es dificil probar que el número $\nu_{i}$ es independiente de la elección de la carta $(U, \phi)$, por esta razón el entero $\nu_{i}$ es llamado el orden de $Z_{i}$ en $p$ y lo denotamos por $\operatorname{ord}_{p}\left(Z_{i}\right)$. La multiplicidad algebraica de la foliación $\mathcal{F}_{\eta}$ (o del campo $Z$ ) en el punto $p \in \mathcal{M}^{n}$, denotada por $m_{p}\left(\mathcal{F}_{Z}\right)$ (o simplemente por $m_{p}(Z)$ ), es definida como el mínimo de los órdenes $\operatorname{ord}_{p}\left(Z_{i}\right)$.

Un punto $p \in \mathcal{M}^{n}$ es llamado punto singular de la foliación $\mathcal{F}_{Z}$ (o del campo $Z$ ) si y sólo si $m_{p}(Z) \geq 1$, en caso contrario decimos que $p$ es un punto regular. El conjunto de todos los puntos singulares de la foliación $\mathcal{F}_{Z}$ será denotado por $\operatorname{Sing}\left(\mathcal{F}_{Z}\right)$. Un punto $p \in \mathcal{M}^{n}$ es llamado singularidad aislada de $Z$ si y sólo si $p \in \operatorname{Sing}\left(\mathcal{F}_{Z}\right)$ y existe una vecindad abierta $U \subseteq \mathcal{M}^{n}$ de $p$ tal que todos los elementos de $U-\{p\}$ son puntos regulares de $Z$. Un punto $p \in \operatorname{Sing}\left(\mathcal{F}_{Z}\right)$ es llamado punto singular reducido si y sólo si $m_{p}(Z)=1$ y la parte lineal de $Z$ en $p$ tiene al menos un autovalor no nulo.

Sea $E: \tilde{\mathcal{M}}^{n} \rightarrow \mathcal{M}^{n}$ el blow-up centrado en el punto $p \in \mathcal{M}^{n}$, singularidad aislada de $\mathcal{F}_{Z}$. Existe una única manera de extender el pull-back $E^{*}\left(\mathcal{F}_{Z_{1}}-\{p\}\right)$ a una foliación analítica singular por curvas, denotada por $\tilde{\mathcal{F}}_{Z}$, la cual está definida en una vecindad del espacio proyectivo $\mathbb{C} P(n-1)=E^{-1}(p) \subset \tilde{\mathcal{M}}^{n}$ y cuyo conjunto singular tiene codimension $\geq 2$. Esta foliación $\tilde{\mathcal{F}}_{7}$ es llamada la transformada estricta de $\mathcal{F}_{Z}$ por $E$ y el espacio proyectivo $E^{-1}(p)$ es llamado divisor. Denotaremos por $\tilde{Z}$ al campo vectorial holomorfo que genera a la foliación $\tilde{\mathcal{F}}_{Z}$. El punto $p \in \mathcal{M}^{n}$ es llamado singularidad no dicrítica de $\mathcal{F}_{Z}$ si y sólo si el divisor es invariante por $\tilde{\mathcal{F}}_{Z}$, esto significa que $E^{-1}(p)$ es la unión de hojas y singularidades de $\tilde{\mathcal{F}}_{Z}$. En caso contrario, decimos que $p$ es una singularidad dicrítica de $\mathcal{F}_{7}$.

El Problema de desingularización para una singularidad (dicrítica o no) de $p \in \mathcal{M}^{n}$ de $\mathcal{F}_{Z}$, consiste en probar la existencia de una función holomorfa propia, $\phi: \mathcal{M}^{*} \rightarrow \mathcal{M}^{n}$ de una variedad compleja $n$-dimensional $\mathcal{M}^{*}$ que cumple las siguientes condiciones:

- $\phi^{-1}(p)=\bigcup_{i=1}^{n} D_{i}$ es unión de subvariedades complejas compactas de codimensión uno y con cruzamientos normales.

- Ta foliación pull-back $\phi^{*}\left(\left.\mathcal{F}_{Z}\right|_{\mathcal{M}^{n}-\{p\}}\right)$ se extiende a una foliación de $\mathcal{M}^{*}$ con conjunto singular de codimensión $\geq 2$ y tal que todos sus puntos singulares son reducidos. 
En dimensión $n=2$, el problema de Desingularización fue estudiado por I. Bendixson [3] y H. Dulac [8] a comienzos del siglo XX, sin embargo, su solución sólo fue dada por A. Seidenberg [12] a finales de la década del sesenta. Posteriormente, A. Ven Den Essen [13] presentó una demostración más geométrica, usando el concepto de multiplicidad de intersección de curvas analíticas complejas.

Para dimensiones mayores que dos, el problema aún sigue abierto. Algunas soluciones parciales han sido dadas por diversos autores, por ejemplo F. Cano [7] establece diversas estrategias para resolver el problema de desingularización en dimensión 3 (sin embargo, un resultado definitivo todavía no se ha encontrado), C. Camacho, F. Cano y P. Sad [6] resuelven el problema en dimensión $n$, bajo la hipótesis que $p$ es una singularidad absolutamente aislada y no dicrítica, generalizando un método dado por C. Camacho y P. Sad en [5] cuando $n=2$. Este resultado fue mejorado por R. Benazic [2], siempre en el caso $n$-dimensional.

En el presente trabajo nosotros resolvemos el problema de desingularización para el caso en que $p$ es una singularidad absolutamente aislada de una variedad compleja de dimensión 3 .

\section{SINGULARIDAD ABSOLUTAMENTE AISLADA}

Una hipótesis natural para resolver el problema de desingularización. es asumir que en cada etapa del blow-up, el conjunto singular de la foliación levantada siempre tenga codimensión $n$, más específicamente, tenemos la siguiente

Definición 1. Sea $\mathcal{F}_{7}$ una foliación analítica por curvas sobre una variedad compleja $n$-dimensional $\mathcal{M}^{n}$. Decimos que $p \in \operatorname{Sing}\left(\mathcal{F}_{Z}\right)$ es una singularidad absolutamente aislada (S.A.A) de $\mathcal{F}_{Z}$ si y sólo si se verifican las siguientes condiciones:

1. $p$ es una singularidad aislada de $\mathcal{F}_{7}$.

2. Denotemos $p=p_{0}, \mathcal{M}^{n}=\mathcal{M}_{0}^{n}, \mathcal{F}_{Z}=\mathcal{F}_{0}, \tilde{\mathcal{M}}^{n}=\mathcal{M}_{1}^{n}, \tilde{\mathcal{F}}_{Z}=\mathcal{F}_{1}$, $E_{1}=E$. Si consideramos una sucesión finita de blow-up's

$$
\mathcal{M}_{0}^{n} \stackrel{E_{1}}{\longleftarrow} \mathcal{M}_{1}^{n} \stackrel{E_{2}}{\longleftarrow} \ldots \stackrel{E_{N}}{\longleftarrow} \mathcal{M}_{N}^{n}
$$

donde el centro de cada $E_{i}$ es un punto $p_{i-1} \in \operatorname{Sing}\left(\mathcal{F}_{i-1}\right)$ (en donde $\mathcal{F}_{j}$ denota el transformado estricto de la foliación $\mathcal{F}_{j-1}$ por $\left.E_{j}, 1 \leq i, j \leq N\right)$, entonces $\operatorname{Sing}\left(\mathcal{F}_{N}\right)$ es un conjunto finito.

Nuestra definición de singularidad absolutamente aislada es más general que la dada en [6]. En esta referencia los autores suponen que 
en cada etapa del blow-up, el transformado estricto sólo presenta singularidades aisladas no dicríticas (este concepto podría ser llamado singularidad absolutamente aislada no dicrítica). En nuestro caso, las singularidades dicríticas pueden aparecer en una o más etapas durante el proceso del blow-up.

El objetivo es probar el siguiente teorema de desingularización:

TEOREMA A. Sea $p \in \mathcal{M}^{n}$ una singularidad absolutamente aislada de $\mathcal{F}_{Z}$. Denotemos $p=p_{0}, \mathcal{M}^{n}=\mathcal{M}_{0}^{n}, \mathcal{F}_{Z}=\mathcal{F}_{0}$ y $E_{1}=$ E. Entonces existe una sucesión finita de blow up's

$$
\mathcal{M}_{0}^{n} \stackrel{E_{1}}{\longleftarrow} \mathcal{M}_{1}^{n} \stackrel{E_{2}}{\longleftarrow} \ldots \stackrel{E_{N}}{\longleftarrow} \mathcal{M}_{N}^{n}
$$

que satisface las siguientes propiedades:

1. El centro de cada $E_{i}$ es un punto $p_{i-1} \in \operatorname{Sing}\left(\mathcal{F}_{i-1}\right)$, donde $\mathcal{F}_{j}$ denota el transformado estricto de la foliación $\mathcal{F}_{j-1}$ por $E_{j}(1 \leq$ $i, j \leq N)$.

2. Si $q \in \operatorname{Sing}\left(\mathcal{F}_{N}\right)$ entonces $q$ es singularidad reducida.

La principal herramienta usada en la prueba del Teorema A es una fórmula que relaciona la multiplicidad algebraica de la singularidad con el número de Milnor de las singularidades que aparecen después de un blow-up.

\section{EL NUMERO DE MILNOR DE UN CAMPO VECTORIAL HOLOMORFO}

Sea $U \subseteq \mathbb{C}^{n}$ un abierto, denotemos por $\mathcal{O}_{n, p}$ al anillo de gérmenes de las funciones analíticas en $p \in U$ y sea $I\left[Z_{1}, Z_{2}, \ldots, Z_{n}\right] \subseteq \mathcal{O}_{n, p}$ el ideal generado por las componentes del campo vectorial holomorfo $Z$ definido en $U$. El Número de Milnor del campo $Z$ en el punto $p$, denotado por $\mu_{p}(Z)$, es definido como

$$
\mu_{p}(Z)=\operatorname{dim}_{\mathbb{C}}\left(\frac{\mathcal{O}_{n, p}}{I\left[Z_{1}, Z_{2}, \ldots, Z_{n}\right]}\right)
$$

Este número satisface las siguientes propiedades (ver [9]):

1. $\mu_{p}(Z)$ es finito si y sólo si $p$ es una singularidad aislada de $Z$.

2. $\mu_{p}(Z)=0$ si y sólo si $p$ es un punto regular de $Z$.

3. $\mu_{p}(Z)=1$ si y sólo si $\operatorname{det}\left(\frac{\partial Z_{i}(p)}{\partial z_{j}}\right)_{1 \leq i, j \leq n} \neq 0$.

Debemos mencionar también que si $p \in \mathcal{M}^{n}$ es una singularidad aislada de $Z$, entonces $\mu_{p}(Z)$ coincide el grado topológico de la función de 
Gauss inducido por $Z$ el cual es considerado como un campo vectorial real, en una esfera de dimension real $2 n-1$, suficientemente pequeña centrada en $p$.

Sean $p \in U$ una singularidad aislada del campo vectorial $Z$, tal que $m_{p}(Z)=\nu, \mathcal{F}_{Z}$ la foliación generada por $Z, \tilde{\mathcal{F}}_{Z}$ el transformado estricto de $\mathcal{F}_{7}$ y $\tilde{Z}$ el campo vectorial holomorfo que genera a la foliación $\tilde{\mathcal{F}}_{7}$. Cuando $n=2$, existe una fórmula que relaciona $\nu$ con el número de Milnor de $Z$ en $p$ y el número de Milnor de las singularidades de $\tilde{Z}$ (ver $[11],[4])$ :

$$
\mu_{p}(Z)-\left\{\begin{array}{l}
\nu^{2}-\nu-1+\sum_{q \in E^{-1}(p)} \mu_{q}(\tilde{Z}), \text { si } p \text { es no dicrítico. } \\
\nu^{2}+\nu-1+\sum_{q \in E^{-1}(p)} \mu_{q}(\tilde{Z}), \text { si } p_{\text {. es dicrilico. }}
\end{array}\right.
$$

Obscrve quc como cl conjunto Sing $\left(\tilde{\mathcal{F}}_{Z}\right)$ cs finito, las sumatorias que aparecen en la expresion anterior son finitas. Existe una generalización $n$-dimensional de la fórmula anterior bajo las hipótesis de que $p$ es una singularidad aislada no dicrítica de $Z$ y el conjunto $\operatorname{Sing}\left(\tilde{\mathcal{F}}_{Z}\right)$ es finito (ver [6]):

$$
\mu_{p}(Z)-\nu^{n}-\nu^{n-1}-\ldots-\nu-1+\sum_{q \in E^{-1}(p)} \mu_{q}(\tilde{Z})
$$

En el caso de singularidades dicríticas en dimension tres, tenemos el siguiente resultado (ver [1])

TEOREMA 1. Sea $Z$ un campo vectorial holomorfo con singularidad aislada en $p \in \mathbb{C}^{3}$, tal que $\tilde{Z}$ tiene singularidades aisladas en el divisor. Si $p \in \mathbb{C}^{3}$ es una singularidad dicrítica de $Z$ y $m_{p}(Z)=\nu$, entonces

$$
\mu_{p}(Z)=\nu^{3}+2 \nu^{2}-2+\sum_{q \in E^{-1}(p)} \mu_{q}(\tilde{Z})
$$

\section{PRUEBA DEL TEOREMA A}

Sea $m_{p}(Z)=\nu \geq 2$. Desde que $p$ es una S.A.A. de $\mathcal{F}_{Z}$, de (3.1) y el Teorema 1 , tenemos que 
$\mu_{p}(Z)= \begin{cases}\nu^{3}-\nu^{2}-\nu-1+\sum_{q \in E^{-1}(p)} \mu_{q}(\tilde{Z}), & \text { si } p \text { es no dicrítico. } \\ \nu^{3}+2 \nu^{2}-2+\sum_{q \in E^{-1}(p)} \mu_{q}(\tilde{Z}), & \text { si } p \text { es dicrílico. }\end{cases}$

En cualquier caso (dicrítico o no) se tiene que

$$
\nu>1 \Longrightarrow \mu_{q}(\tilde{Z})<\mu_{p}(Z), \forall q \in E^{-1}(p)
$$

Además, se cumple

$$
\nu \leq \mu_{p}(Z), \forall p
$$

Afirmo que después de un número finito de blow-up's $E_{1}=E$, $E_{2}, \ldots, E_{N}$ con centro en puntos singulares, se obtiene solamente puntos con multiplicidad algebraica $\leq 1$. En efecto, caso contrario, en cada blow-up existiría por lo menos un punto en el divisor cuya multiplicidad algebraica es $\geq 2$, i.e.

$$
\text { 1)ado } k \in \mathbb{N}, \exists p_{k} \in H^{-1}\left(p_{k-1}\right) \text { tal que } m_{p}\left(/^{(k)}\right) \geq 2 \text {, }
$$

en donde $Z^{(1)}-\tilde{Z}$ y $Z^{(k)}-\tilde{Z}^{(k-1)}$. De (4.1) se sigue que

$$
\begin{aligned}
& \mu_{p_{1}}\left(Z^{(1)}\right)<\mu_{p}(Z), \mu_{p_{2}}\left(Z^{(2)}\right)<\mu_{p_{1}}\left(Z^{(1)}\right), \ldots \\
& \ldots, \mu_{p_{k}}\left(Z^{(k)}\right)<\mu_{p_{k-1}}\left(Z^{(k-1)}\right), \ldots \quad \forall k \in \mathbb{N}
\end{aligned}
$$

lo cual implica que

$$
\mu_{p}(Z)>\mu_{p_{1}}\left(Z^{(1)}\right)>\ldots>\mu_{p_{k}}\left(Z^{(k)}\right)>\ldots \geq 1
$$

lo cual es, evidentemente, una contradicción. Esto prueba la afirmación.

Definimos $\varphi=E_{N} \circ E_{N-1} \circ \ldots \circ E$, se sigue que $\varphi: \mathcal{M}_{N}^{3} \rightarrow \mathcal{M}_{0}^{3}$ cs una función holomorfa propia y cl pull-back $\phi^{*}\left(\left.\mathcal{F}_{0}\right|_{\mathcal{M}^{3}-\{p\}}\right)$ sc cxtiende a una foliación singular $\mathcal{F}_{N}$ sobre $\mathcal{M}_{N}^{3}$ con conjunto singular de codimensión 3.

Por lo tanto, si $q \in \operatorname{Sing}\left(\mathcal{F}_{N}\right)$ entonces $m_{q}\left(\mathcal{F}_{N}\right)=1$. Por la forma canónica de Jordan, $q$ no es una singularidad reducida si la parte lineal del campo vectorial holomorfo que genera $\mathcal{F}_{N}$ es de la forma 


$$
\left(\begin{array}{lll}
0 & 1 & 0 \\
0 & 0 & 0 \\
0 & 0 & 0
\end{array}\right) \quad \text { ó }\left(\begin{array}{lll}
0 & 1 & 0 \\
0 & 0 & 1 \\
0 & 0 & 0
\end{array}\right)
$$

El Teorema A es una consecuencia del siguiente

LEMA. Sea $Z$ un campo vectorial holomorfo con singularidad aislada en $0 \in \mathbb{C}^{3}$, tal que $m_{0}(Z)=1$. Si $D Z(0)$ es de la forma

$$
\text { a) }\left(\begin{array}{lll}
0 & 1 & 0 \\
0 & 0 & 0 \\
0 & 0 & 0
\end{array}\right) \text { ó }\left(\begin{array}{lll}
0 & 1 & 0 \\
0 & 0 & 1 \\
0 & 0 & 0
\end{array}\right) \text {, }
$$

entonces 0 no es una S.A.A.

\section{Prueba del Lema.}

a) En este caso, el campo vectorial es de la format

$$
\begin{aligned}
Z(x, y, z)= & \left(y+\sum_{k \geq 2} A_{k}(x, y, z)\right) \frac{\partial}{\partial x}+\left(\sum_{k \geq 2} B_{k}(x, y, z)\right) \frac{\partial}{\partial y} \\
& +\left(\sum_{k \geq 2} C_{k}(x, y, z)\right) \frac{\partial}{\partial z}
\end{aligned}
$$

En la carta del blow-up $t-\frac{y}{x}, s-\frac{z}{x}$, un simple cálculo muestra que el transformado estricto $\tilde{\mathcal{F}}_{Z}$ es generado por:

$$
\begin{aligned}
\tilde{Z}(x, t, s)= & x\left(t+A^{*}(x, t, s)\right) \frac{\partial}{\partial x}+\left(-t^{2}+x B^{*}(x, t, s)\right) \frac{\partial}{\partial t} \\
& +\left(-t s+C^{*}(x, t, s)\right) \frac{\partial}{\partial s}
\end{aligned}
$$

Por lo tanto, es fácil ver que cualquier punto de la forma $(0,0, s)$, con $s \in \mathbb{C}$ cs un punto singular de $\tilde{\mathcal{F}}_{Z}$. Sc siguc que $\operatorname{Sing}\left(\tilde{\mathcal{F}}_{Z}\right)$ ticnc infinitos elementos y, por lo tanto, 0 no es una S.A.A. de $\mathcal{F}_{Z}$.

b) En este caso, tenemos que

$$
\begin{aligned}
Z(x, y, z)= & \left(y+\sum_{k \geq 2} A_{k}(x, y, z)\right) \frac{\partial}{\partial x}+\left(z+\sum_{k \geq 2} B_{k}(x, y, z)\right) \frac{\partial}{\partial y} \\
& +\left(\sum_{k \geq 2} C_{k}(x, y, z)\right) \frac{\partial}{\partial z}
\end{aligned}
$$


Es fácil ver que cl $(0,0,0)$ cn la carta $t=\frac{y}{x}, s=\frac{z}{x}$ cs la única singularidad de $\tilde{\mathcal{F}}_{Z}$, más aún, un simple cálculo nos muestra que $\tilde{\mathcal{F}}_{Z}$ es generado por

$$
\tilde{Z}=\tilde{A} \frac{\partial}{\partial x}+\tilde{B} \frac{\partial}{\partial t}+\tilde{C} \frac{\partial}{\partial s}
$$

donde

$$
\mid \begin{aligned}
& \tilde{A}(x, t, s)-x t+\sum_{k \geq 2} A_{k}(1, t, s) x^{k} \\
& \tilde{B}(x, t, s)-s-t^{2}+\sum_{k \geq 2}\left[B_{k}(1, t, s)-t A_{k}(1, t, s)\right] x^{k-1} \\
& \tilde{C}(x, t, s)=-t s+\sum_{k \geq 2}\left[C_{k}(1, t, s)-s A_{k}(1, t, s)\right] x^{k-1}
\end{aligned}
$$

No es difícil ver que

$$
D \tilde{Z}(0)=\left(\begin{array}{lll}
0 & 0 & 0 \\
\alpha & 0 & 1 \\
\beta & 0 & 0
\end{array}\right)
$$

donde $\alpha=B_{2}(1,0,0)$ y $\beta=C_{2}(1,0,0)$. Se sigue que $(0,0,0)$ es también un punto singular no reducido de $\tilde{\mathcal{F}}_{7}$. Distinguimos dos casos:

CASO 1: $\beta-0$. En la carla $t^{\prime}-\frac{l}{x}, s^{\prime}-\frac{s}{x}$, el transformado estricto de $\tilde{\mathcal{F}}_{7}$ es generado por:

$$
Z^{(2)}\left(x, t^{\prime}, s^{\prime}\right)=\left(\alpha+s^{\prime}\right) \frac{\partial}{\partial t^{\prime}}+\tilde{Y}\left(x, t^{\prime}, s^{\prime}\right)
$$

donde $\tilde{Y}$ es un campo vectorial holomorfo. Por lo tanto, cualquier punto de la forma $\left(0, t^{\prime},-\alpha\right)$ con $t^{\prime} \in \mathbb{C}$ es un punto no singular de $\tilde{\mathcal{F}}_{Z}^{(2)}$, sc siguc que $\operatorname{Sing}\left(\tilde{\mathcal{F}}_{Z}^{(2)}\right)$ cs un conjunto infinito y, por lo tanto, $(0,0,0)$ no es una S.A.A. de $\mathcal{F}_{Z}$.

CASO 2: $\beta \neq 0$. Afirmo que existe un cambio de coordenadas lineal $L$ tal que $L^{*} \tilde{Z}$ satisface las condiciones del caso 1 . En efecto, definimos las transformaciones lineales $L, T: \mathbb{C}^{3} \rightarrow \mathbb{C}^{3}$ como $L\left(z_{1}, z_{2}, z_{3}\right)=$ $\left(\frac{1}{\beta} z_{3}, z_{1}, z_{2}-\frac{\alpha}{\beta} z_{3}\right)$ y ' ' $\left(w_{1}, w_{2}, w_{3}\right)=\left(w_{2}, \alpha w_{1}+w_{3}, \beta w_{1}\right)$. E's claro que $T=L^{-1}$. Defino

$$
X=L^{*} \tilde{Z}=T \tilde{Z} \circ L=A^{*} \frac{\partial}{\partial x_{1}}+B^{*} \frac{\partial}{\partial x_{2}}+C^{*} \frac{\partial}{\partial x_{3}},
$$

donde $A^{*}=\tilde{B} \circ L, B^{*}=\alpha \tilde{A} \circ L+\tilde{C} \circ L$ y $C^{*}=\beta \tilde{A} \circ L$. Observe que: 


$$
D X(0)=T \cdot D \check{Z}(0) \cdot L=\left(\begin{array}{lll}
0 & 1 & 0 \\
0 & 0 & 1 \\
0 & 0 & 0
\end{array}\right)
$$

y $C_{2}^{*}(1,0,0)=0$. Por lo tanto, 0 no es una S.A.A. de $X$ y de este modo, el Lema esta probado.

\section{BIBLIOGRAFÍA}

[1] R. Benazic, Sobre el número de Milnor, PESQUIMAT, Vol. III, N 1, (2000), p. 1-15.

[2] R. Benazic, $A$ resolution theorem for absolutely isolated singularities of holomorphic vector fields, Bol. Soc. Bras. Mat., Vol 28, N 1, (1997), p. 211-231.

[3] I. Bendixson, Sur les points singuliers des équations singuliers des équations differentielles, Ofv. Kongl. Vetenskaps Akademiens Förhandlinger, Stokholm, Vol 9, 186, (1898), p. 635-658.

[4] C. Camacho, Holomorphic Dynamical Systems, Summer School on Dynamical Systems, ICTP, Trieste - Italia, (16 August - 9 September, 1988).

[5] C. Camacho, P. Sad, Pontos singulares de Equações Diferenciais Analíticas, $16^{\circ}$ Colóquio Brasileiro de Matemática, IMPA, (1987).

[6] C. Camacho, F. Cano, P. Sad, Absolutely isolated singularities of holomorphic vector fields, Invent. math. 98, (1989), p. 351-369.

[7] F. Cano, Desingularization strategies for 3-dimensional vector fields, Lecture Notes in Math., Vol. 1259. Berlin, Heidelberg, New York; Springer(1987).

[8] H. Dulac, Recherches sur les points singuliers des équations différentielles, J. École Polytechnique, Vol 2, sec. 9, (1904), p. 1-125.

[9] P. Griffiths, J. Harris, Principles of Algebraic Geometry, Wiley-Intersciencie, New York, (1978).

[10] R. Gunning, H. Rossi, Analytic functions of several complex variables, PrenticeHall, Englewood Cliffs, NJ., (1965).

[11] J. Mattei, R. Moussu, Holonomie et intégrales premières, Ann. Sci. Ecole. Norm. Sup. (4) 13 (1980) p. 469-523.

[12] A. Seidenberg, Reduction of singularities of the differentiable equation Ady $=$ Bdx, Am. J. Math. 90 (1968) p. 248-269.

[13] A. Ven Den Essen, Reduction of singularities of the differentiable equation Ady $=B d x$ Lecture Notes in Math., Vol. 712 p. 44-59, Springer-Verlag.

[14] H. Whitney, Complex Analytic Varieties, Addison-Wesley Publishing Company, (1972). 